\title{
ON THE NATURE OF RYLE AND BAILEY'S CANDIDATE STAR FOR THE PULSATING RADIO SOURCE CP 1919
}

\author{
J. A. Westphal, Jerome Kristian, Grant Snellen, Allan Sandage, \\ MaArten Schmidt, J. B. Oke, Gerry Neugebauer, \\ AND E. E. BECKLIN \\ Mount Wilson and Palomar Observatories, Carnegie Institution of Washington, \\ California Institute of Technology \\ Received December 16, 1968
}

\section{ABSTRACT}

The energy distribution and spectrum of the "blue" star near CP 1919 indicate that it is a normal early $\mathrm{F}$, main-sequence star and therefore is not likely to be related to the radio source. No regular variations were found in the light from the star, to within an accuracy of a few tenths of 1 per cent.

Shortly after the announcement by Hewish et al. (1968) of the discovery of the pulsating radio sources, Ryle and Bailey (1968) suggested a possible identification of the source CP 1919 with a star at $a=19^{\mathrm{h}} 19^{\mathrm{m}} 36^{\mathrm{s}} .9, \delta=+21^{\circ} 46^{\prime} 57^{\prime \prime}$ (1950), based on coincidence with the best available radio position.

This Letter reports the results of photoelectric monitoring, broad-band photometry, and spectral scans of this star. These measurements, together with the spectroscopic data, show that it is a normal main-sequence star of spectral type early $F$, which would mean that it is not likely to be associated with the pulsating radio source.

We have attempted several times to detect light variations of the star, using the 200inch prime-focus photometer and special electronic equipment. Table 1 lists the pertinent data for the observations; the diaphragm was $8^{\prime \prime}$ in diameter. Figure 1 shows the electronics arrangement at the telescope. Pulses from the photomultiplier were shaped and amplified and then applied to the scaling input of a 1024-channel signal-averaging computer set at a sweep time of $16 \mathrm{sec}$. The averager is a core storage device which accumulates pulse counts for $\frac{16}{10 \frac{6}{24}} \mathrm{sec}$ in each successive channel. A new sweep was initiated every 12 radio periods of the pulsating radio source (except for the run of May 18/19) as corrected for the motion and rotation of the Earth. This was typically every 16.05 $\mathrm{sec}$, so that there was a dead time of about $50 \mathrm{msec}$ per sweep before the succeeding sweep was started. The trigger pulses for starting the sweeps were obtained by applying a $12-\mathrm{kHz}$ crystal-controlled signal from the 200 -inch data system to a preset digital scaler. Because of the finite frequency, the triggering pulses were systematically out of phase with the radio period by some tens of microseconds per sweep. This phase slip was cumulative over each run and sets a minimum value, of the order of tens of milliseconds, to the detectable pulse widths, in the sense that pulsed variations in the star's light narrower than the minimum value would be widened by this amount and their amplitude decreased proportionately. One would still expect, however, that pulses as wide as the radio pulses would be detected. Values of the total phase slip for each run are included in Table 1 . The accumulated counts in the signal averager were also continuously monitored on an oscilloscope as the data were being taken.

In addition to being averaged at 12 radio periods, the data pulses from the photometer were also recorded directly on magnetic tape, together with the $12-\mathrm{kHz}$ clock signal on a parallel channel of the tape and, for the later runs, the time signal from radio station WWV.

All of the equipment shown in Figure 1 was not used for all of the monitoring runs. In particular, on some nights either the magnetic tape recorder or the signal averager alone was used. The data of May 28/29, obtained only on magnetic tape, were later 
TABLE 1

MONITORING OBSERVATIONS OF THE CANDIDATE STAR FOR CP 1919 WITH THE 200-INCH TELESCOPE

\begin{tabular}{|c|c|c|c|c|c|}
\hline \multirow{2}{*}{$\begin{array}{l}\text { DATE } \\
(1968)\end{array}$} & \multirow{2}{*}{$\begin{array}{c}\text { TOtal } \\
\text { ObSERVING } \\
\text { TIME } \\
\text { (min) }\end{array}$} & \multirow{2}{*}{$\begin{array}{c}\text { Total } \\
\text { Prase } \\
\text { SLIT } \\
\text { (msec) }\end{array}$} & \multicolumn{2}{|c|}{$\begin{array}{l}\text { StaTISTICAL Fluctua- } \\
\text { TIONS (Fraction OF } \\
\text { SigNAL From tHE Star) }\end{array}$} & \multirow{2}{*}{ CoMments } \\
\hline & & & $\begin{array}{c}\text { Per } \\
\text { 16-msec } \\
\text { Channel }\end{array}$ & $\begin{array}{l}\text { Quasi- } \\
\text { sinusoidal } \\
\text { Variations }\end{array}$ & \\
\hline $\begin{array}{l}\text { May } 18 / 19 \text {. } \\
\text { May } 24 / 25 . \\
\text { May 28/29. } \\
\text { August } 2 / 3 \text {. } \\
\text { August } 3 / 4 \text {. }\end{array}$ & $\begin{array}{r}60 \\
70 \\
110 \\
130 \\
70\end{array}$ & $\begin{array}{l}80 \\
60 \\
30 \\
35 \\
15\end{array}$ & $\begin{array}{l}4 \% \\
3 \\
2 \\
3 \\
3 \%\end{array}$ & $\begin{array}{l}0.2 \% \\
.1 \\
.15 \\
0.15 \%\end{array}$ & $\begin{array}{l}\text { Averaged at } 6 \text { radio periods } \\
\text { Raw data only } \\
\text { V-band }\end{array}$ \\
\hline
\end{tabular}

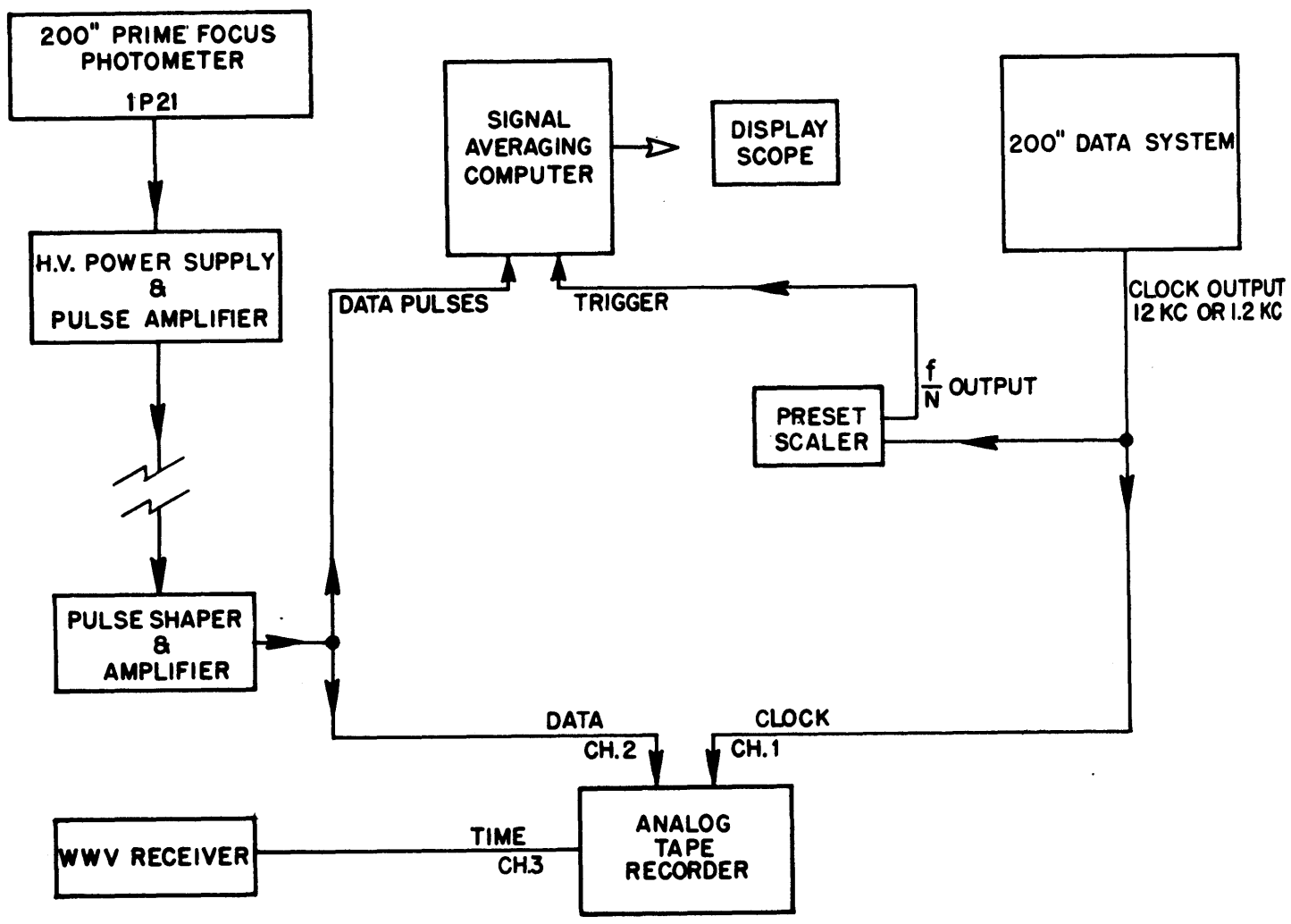

Fig. 1.-Electronics arrangement designed to detect rapid, regular light variations 
played back through the signal averager in the laboratory, with an electronics arrangement similar to that at the telescope. A "flywheel" oscillator synchronized to the clock signal from the tape was added to fill in dropouts from the clock track due to chips and dirt on the surface of the tape. It is essential to take the clock signal from the tape itself in order to insure that the averager sweeps are properly phased in spite of tape-speed variations. There was still a small residual smearing of the data due to variations of tape drive speed within each sweep, since the averager advances channels in real time, but the effect is not cumulative and was found to be negligible compared with the phase slip due to the finite clock frequency.

Figure 2, $a$, shows the data for the run of August 2/3. This is a point-by-point plot of the 1024 channels of the signal averager, as recorded at the telescope at 12 radio periods. The scatter about the mean is about 3 per cent of the signal from the star, which is consistent with the expected statistical fluctuations for the measured photon counting rate. To within this accuracy, no fluctuations in the light from the star are seen at 12 radio periods or any submultiple of 12 periods.

To improve the accuracy, the accumulated counts in the signal averager were further processed by digital computer. The 1024 channels were divided into groups equivalent to $6,4,3,2$, and 1 radio periods and the groups added to produce equivalent records at those periods. The data for each period were then smoothed by numerical Gaussian filters with equivalent widths varying between the minimum detectable pulse width, as determined from the total phase slip in each case, to about 40 per cent of the total period, the latter to detect any possible quasi-sinusoidal variations. Table 1 includes the statistical fluctuations for the extreme cases in each run, viz., (a) fluctuations per 16-msec channel of the averager and (b) quasi-sinusoidal variations.

No variations were found in any of our data at any pulse width or period. In particular, the quasi-sinusoidal variations at 2 radio periods suggested earlier (Lynds, Maran, and Trumbo 1968) are not observed, although we would have detected a variation with an amplitude an order of magnitude smaller than the suggested amplitude of several per cent.

Finally, in order to search for fluctuations of the star at arbitrary frequencies, we computed power spectra for the tape-recorded data from the best three runs, those of May 28/29, August 2/3, and August 3/4. To avoid difficulties due to tape-speed variations, the data samples for the analysis were obtained by digitally integrating the data pulses for successive $100-\mathrm{msec}$ intervals, determined by digitally dividing the $12-\mathrm{kHz}$ clock signal from the tape. The power spectra obtained were those of random noise.

As a check on the experiment, we used the same equipment and techniques to study the fastest known regular stellar variation-the 71-sec period of DQ Her, discovered by Walker (1956). This was done on August 3/4, just before the run on the pulsating-radiosource candidate. The only changes made were prescaling the clock from 12 to $1.2 \mathrm{kHz}$ in order to accommodate the longer period, and changing the sweep time of the averager from 16 to $128 \mathrm{sec}$. Sweeps were started every $142.13 \mathrm{sec}$, which is twice the period of the star. The 71-sec variation could be discerned on the oscillscoope after three sweeps and continued to build uniformly as expected over the 40 minutes of observing. Figure $2, b$, shows the raw data as recovered from the averager. The amplitude of the variation is about 2 per cent of the signal from the star, which happens also to be nearly the scatter per channel due to photon statistics. Since we counted for only $128 \mathrm{sec}$ out of every 2 periods, the curve shows only 1.8 periods. The variation is quite apparent in the raw data, with no further reduction.

In order to test our method of recovering data from magnetic tape, we simultaneously recorded this run and reduced it, using the same methods as for the pulsating-radiosource candidate, and again recovered the light curve of the star. Finally, we computed the power spectrum of the taped data and found the expected peak at $\frac{1}{71} \mathrm{~Hz}$. The data for DQ Her are quite interesting in themselves and will be discussed in detail elsewhere. 
$10 \%-$

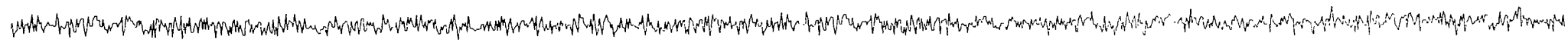

$10 \%-$

(a)

DQ Her

$10 \%-$

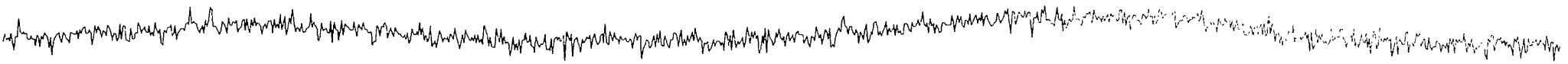
$-10 \%-$

I

(b)

Fig. 2.-Monitoring runs on the pulsating-radio-source candidate and DQ Her obtained with the electronics arrangement shown in Fig. 1. Amplitude scales are expressed as fractions of the light from the stars. These are point-by-point plots of the 1024 data channels of the signal averager as recorded at the telescope, averaged at 12 radio periods for CP 1919 and two 71-sec periods for DQ Her. 
We believe that these tests show that the system works as it should and that we would have detected variations in the pulsating-radio-source candidate had they occurred.

In sum, we have detected no regular variations of Ryle and Bailey's candidate star, to within an accuracy as high as a few tenths of 1 per cent for quasi-sinusoidal variations at multiples of the radio period, and from our data we have no reason to believe that the star has any regular variations on a 1 -sec time scale.

In view of this negative result, we must ask whether the candidate star is likely to be related to the pulsating radio source. An independent measurement of the radio position of CP 1919 by Turtle and Vaughan (1968) and a revision of the Cambridge position suggest that the radio source lies about $5^{\prime \prime}$ farther to the northeast than originally thought, making less plausible an identification based on the coincidence of positions.

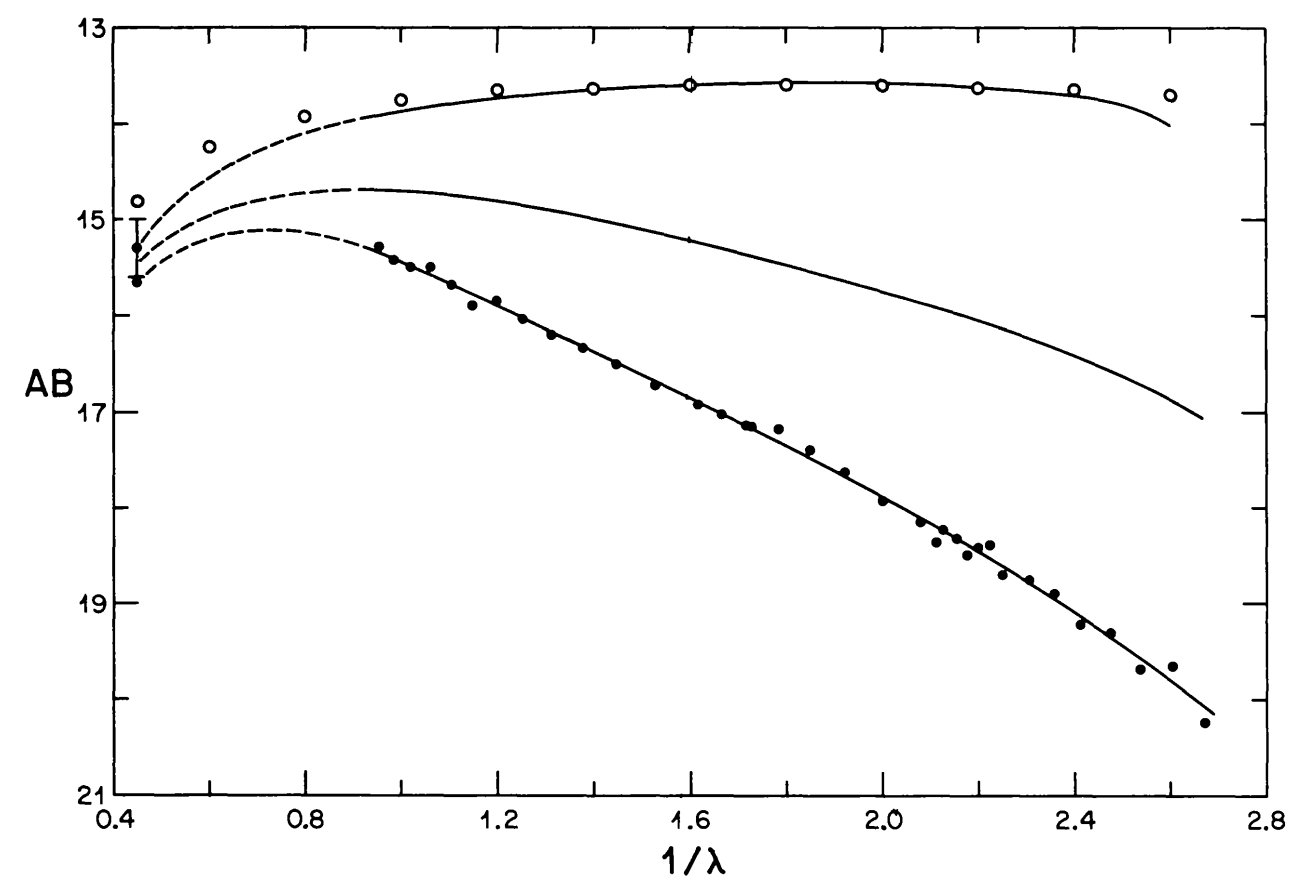

FIG. 3.-Absolute spectral-energy distribution $\mathrm{AB}=-2.5 \log f_{\nu}-48.55$, where $f_{\nu}$ is the flux in ergs $\mathrm{cm}^{-2} \mathrm{sec}^{-1} \mathrm{~Hz}^{-1}$ for the star near the pulsating radio source CP 1919. Plotted points are observed fluxes. Upper curves have been derived from the curve through the points by removing interstellar reddening corresponding to total absorptions at $5556 \AA$ of 1.9 and $3.8 \mathrm{mag}$. Open circles represent a model atmosphere with $T_{e}=7750^{\circ} \mathrm{K}$ and $\log g=4.44$. The standard deviation of the point at $1 / \lambda=0.44$ is shown for the uppermost curve.

Lynds (Lynds et al. 1968) has obtained several spectrograms of the star, on the basis of which he classifies it as A7-F0. We have obtained $U B V$ photometry of the candidate star and thirteen other stars in the field. The measurements for the candidate are $V=$ 17.57, $B-V=+1.40$, and $U-B=+0.84$. These are consistent with the star's being an F0 main-sequence star at a distance of $1800 \mathrm{pc}$, with reddening due to interstellar absorption of $E(B-V)=1.10 \mathrm{mag}$. This amount of reddening is, in turn, consistent with the measurements of other stars in the field.

The above conclusions are supported by detailed photoelectric scans and infrared photometry. The absolute spectral-energy distribution was obtained in the blue using the prime-focus spectrum scanner on the 200-inch Hale telescope and in the wavelength interval 5500-10500 $\AA$ with the new multichannel photoelectric spectrometer. The fluxes are based on the absolute calibration of a Lyr given by Oke (1964). The band pass was 100 or $200 \AA$ in the blue and $360 \AA$ in the red and near-infrared. One further point at $2.2 \mu$ was obtained with a broad-band photometer on two nights. The results 
are plotted as solid dots in Figure 3. The standard deviation is in almost every case less than $0.05 \mathrm{mag}$, except for the $2.2-\mu$ point, where it is 0.3 mag. The smoothed energy distribution was corrected for reddening by using Whitford's reddening curve (Whitford 1958). The two upper curves show the corrected results for total interstellar absorption at $5556 \AA$ of 1.9 and $3.8 \mathrm{mag}$, respectively. The open circles represent a model atmosphere with $T_{e}=7750^{\circ} \mathrm{K}$ and $\log g=4.44$. The fit to the uppermost reddening curve is within the accuracy of the observations, and there is no reason to suppose that the object is not an ordinary star of spectral type near F0.

On the weight of the evidence, we conclude that the most likely possibility is that the candidate star is a normal main-sequence star, unrelated to the pulsating radio source. If this is true, then an upper limit of about 22 mag (photographic) can be set for the optical brightness of the pulsating radio source from Arp's 200-inch plate of the field, which shows no stars brighter than this magnitude within the error boxes of the radio position (a print of this plate is shown in Cameron and Maran 1968). A similar limit been set for CP 0950 and CP 1133 (Kristian 1968).

We are indebted to W. L. W. Sargent for a discussion of the spectroscopic data and to $\mathbf{M}$. Ryle for furnishing a revised Cambridge position. This research was supported in part by NASA grant NGL 05-002-007 and ARPA grant DA-ARO-D-31-124-G 705.

\section{REFERENCES}

Cameron, A. G. W., and Maran, S. P. 1968, Sky and Tel., 36, 4.

Hewish, A., Bell, S. J., Pilkington, J. D. H., Scott, P. F., and Collins, R. A. 1968, Nature, $217,709$.

Kristian, Jerome. 1968, Ap. J. (Letters), 154, L99.

Lynds, R., Maran, S. P., and Trumbo, D. E. 1968, Science, 161, 42.

Oke, J. B. 1964, Ap. J., 140, 689.

Ryle, M., and Bailey, J. A. 1968, Nature, 217, 907.

Turtle, A. J., and Vaughan, A. E. 1968, Nature, 219, 1845.

Walker, Merle F. 1956, $A p$. J., 123, 68.

Whitford, A. E. 1958, A.J., 63, 201. 